\title{
LA POLÉMICA SOBRE LA CUANTIFICACION EN LAS CIENCIAS DEL HOMBRE
}

\author{
Felipe Martínez Rizo \\ Universidad A. de Aguascalientes
}

Resumen

El articulo presenta un seguimiento de la polémica existente entre la perspectiva analítica cuantitativista y la cualitativista. El autor estructura esta polémica a lo largo del desarrollo cronologico de la metodología de las ciencias sociales y más concretamente en el seno de la sociología. Desde el siglo XVII hasta nuestros dias se va reconstruyendo el desarrollo de la metodologin analitica de la realidad social desde ambas perspectivas, señalándose las aportaciones y los avances de cada una de ellas. Se ofrece un repaso de todos los autores que de un modo u otro han contribuido a ello, asi como de las diferentes escuelas de pensamiento que se formaron al hilo de esta confrontación metodológica.

Resum

L'article presenta un seguiment de la polemica existent entre la perspectiva analítica quantitativista i la qualitativista. L'autor estructura aquesta polemica al llarg del desenvolupament cronològic de la metodologia de les ciencies socials, i més concretament de la metodologia sociologica. Des del segle XVII fins avti, es va reconstruint el desenvolupament de la metodologia analitica de la societat des d'ambdues perspectives, i s'assenyalen les aportacions $i$ els avenços teòrics $i$ practics de cadascuna d'elles. S'ofereix un repàs de tots els autors que d'una manera o d'una altra hi han contribuit, com també de les diferents escoles de pensament que es formen al voltant d'aquesta confrontació de mètode.

\section{INTRODUCCION}

Aunque las polémicas entre partidartos de técnicas cuantitativas y defensores de acercamientos cualitativos en cl ámbito de las ciencias del hombre tie- 
nen ya una larga historia, en la actualidad asistimos a una fase que algunos consideran particularmente aguda de tal enfrentamiento.

La expresión con la que se designa este proceso es la de uguerra de paradigmas", a partir del ambiguo y popular término introducido por Thomas $S$. Kuhn hace casi treinta años.

El 29 de marzo de 1989, en el discurso que pronunció durante el congreso anual de la Asociación Americana de Investigación Educativa (AERA), con motivo de habérsele concedido el premio por aportaciones distinguidas a la investigación, N.L. Gage presentó una visión muy personal de lo que sucedería en los veinte años siguientes, en la forma de una fingida retrospectiva histórica, suponiendo que ya habíamos Ilegado al año 2009.

$\mathrm{La}$ intervención de Gage comenzaba con estas palabras:

Al comenzar a relatar esta historia hemos llegado al año 2009, una década despues del milenio, y estamos mirando hacia atrás para ver qué pasó con la investigación sobre enseñanza en las dos décadas transcurridas desde 1989. ¿Por qué seleccioné esta última fecha como punto de partida de mi retrospección histórica? Porque fue en ese año de 1989 cuando lo que llegó a conocerse como la "guerra de los paradigmas" habia llegado a un sanguinario clímax $(1989$, p. 4).

Gage se refirió a la situación prevaleciente durante la década de los ochenta como antecedente de la supuesta historia de lo que pasaría después, de 1989 a 2009. Y recuerda que el tipo de investigación que había florecido durante los sesenta y los setenta, etiquetada luego como "objetivista-cuantitativista", era cuestionada cada vez con más severidad desde diversas perspectivas que se presentaban a si mismas como alternativas: el punto de vista "antinaturalista", que consideraba sin más que el enfoque científico, adecuado para el estudio de los fenómenos naturales, no lo era en absoluto para las realidades humanas y sociales. El punto de vista "interpretativo", partidario de un acercamiento hermenéutico capaz de comprender sentido e intenciones, más que explicar relaciones causales; y el punto de vista "crítico", que cuestionaba la falta de compromiso político de los investigadores convencionales y su inconsciencia de los supuestos ideológicos de sus trabajos.

Pero las polémicas entre cuantitarivistas y cualitativistas se remontan mucho más atrás, $y$ han conocido otros clímax.

En la actualidad es clato que esta oposición "cuantitativo vs. cualitativo" no representa adectadamente las diversas posturas epistemológicas, que tienen relaciones mucho más complejas y matizadas con el tema preciso de la cuantificación; sin embargo, también parece que no hay un tema particular que haya expresado en forma más condensada la polémica que éste, por lo que en las páginas siguientes se utilizará esta oposición como una especie de abrevia- 
tura para designar las diversas manifestaciones históricas de un enfrentamiento secular entre estilos de investigación muy contrastados.

De todas formas, si esta abreviatura no es del todo adecuada, tampoco lo son - y tal vez mucho menos- otras frecuentemente utilizadas como la oposición «investigación convencional vs. alternativa", «investigación dominante vs. emergente", o "corrientes positivistas vs. postpositivistas".

Con la designación que se prefiera, rastrear la historia de estos enfrentamientos puede ayudar a entenderlos mejor, $y$ a tener una perspectiva más amplia sobre lo que podrá pasar en el futuro, tema al que regresaremos al final de este trabajo para comentar nuevamente la opinión de Gage.

\section{LA "ARITMÉTICA POLITICA" VS. LA "ESTADISTTCA" UNIVERSITARIA EN LOS SIGLOS XVII Y XVIII}

Las ciencias sociales modernas son hijas del siglo XIX, pero su gestación se remonta a bastante más atrás.

Uno de sus antecendentes importantes lo constituyen los progresivos esfuerzos por aplicar procedimientos cuantitativos al estudio de los fenómenos humanos y sociales.

En sus "Notas sobre la historia de la cuantificación en sociología" (1970), Lazarsfeld nos recuerda que los datos cuantitativos más antiguos en ciencias del hombre son demográficos, lo que no parece sorprendente: hace muchos siglos los chinos levantaban ya censos de gran cobertura y precisión.

Luchando con los obstáculos que representaban la reticencia de la población a proporcionar información por temor a los aumentos de impuestos, y el secreto que imponían los gobiernos por considerar que la información demográfica tenía un gran valor militar, para mediados del siglo XVII estaba naciendo la demografía moderna.

Se considera generalmente que su padre fue John Graunt (1620-1674), un londinense que estudiaba los registros mortuorios de las parroquias de Londres, de 1532 en adelante, analizando la frecuencia de las diversas causas de mortalidad, y llegando a la elaboración de las primeras tablas, en las que, en forma muy rudimentaria, a partir de las tasas de supervivencia de dos edades solamente (seis y setenta y seis años), calculó las expectativas de vida de la población.

Graunt publicó sus resultados en 1662, en la obra Observaciones naturales y politicas... hechas a partir de los certificados de defunción.

La obra tuvo éxito y fue reeditada varias veces. La quinta edición fue publicada en 1676, después de la muerte de Graunt, por un buen amigo suyo: William Petty (1623-1687). 
Fue precisamente Petty el creador de una expresión con la que, hasta épocas muy recientes, se designaron en algunos medios los esfuerzos por cuantificar los fenómenos sociales: aritmética politica.

Lazarsfeld presenta lo que podemos considerar el primer episodio de la pelea entre cuantitativistas y cualitativistas, que enfrentó ya entonces a ingleses y alemanes, con el triunfo de los primeros.

Para entender mejor los términos del enfrentamiento recordemos que el sentido original de la palabra "político" no es el que se da actualmente al término, sino que coincide más bien con el sentido actual de la palabra "social».

A partir de esto podemos entender el título de una de las obras importantes de W. Petty, su Anatomía politica de Irlanda (1672).

Petty se refrere al gran abuelo del empirismo inglés, Sir Francis Bacon (1561-1626), quien:

[...] en su "Advancement of Learning" hizo juiciosos paralelismos en numerosos aspecros enrte el cuerpo natural y el cuerpo político, y sobre el arte de conservar ambos en estado de salud y de fuerza; y así como la anatomía es el mejor fundamento en el primer caso, así también lo es en el segundo. Ocuparse de politica sin conocer sus bases es tan inúril como las ocupaciones de las vicjas o los charlatanes (citado por Lazarsfeld, 1970, p. 87).

Hacer anatomia política significaba pues, para Petry, estudiar rigurosamente la estructura del organismo social. Hoy diríamos estudiar científicamente la sociedad, o hacer investigación sociológica, económica, demográfica, antropológica, politológica, etc.

Y hacer "aritmética política» significaba para él estudiar al organismo social utilizando números, cantidades, cifras. Hoy diríamos hacer investigación sociológica, etc., de enfoque cuantitativo.

Petty, y con él la tradición anglosajona, tomó decididamente esta dirección, y concibió el estudio de la sociedad en forma cuantitativa. En sus términos, hizo de la "anatomía política" una "aritmética política", estableciendo una tradición que todavía cultivaban los demógrafos ingleses en la primera mitad del siglo XX, y que influyó directamente en la corriente aún vigente designada con la expresión "empirismo metodológico".

Pero, por la misma época, y partiendo de una inquietud semejante, se desarrolló otro enfoque que hoy podemos designar con el término de cualitativo.

Lazarsfeld explica cómo en 1886 August Meitzen, de la Universidad de Berlín, propuso la tesis de que la estadística moderna surgió de dos fuentes: la aritmética política inglesa y la estadística universitaria alemana; ésta última pretendía describir en forma detallada y exhaustiva, pero no cuantitativa, todos los aspectos posibles de un Estado, y no sólo aquellos que fueran fácilmente cuantificables, como los demográficos (1970, p.85). 
La contraposición actual entre encuesta o survey cuantitativa y extensiva, más superficial, versus estudio de caso, cualitativo e intensivo, más profundo, se encuentra, pues, desde hace mucho tiempo.

Meitzen consideraba que el iniciador de la tradición "cualitativista" alemana había sido un profesor de Gotinga liamado Gottfried Achenwall (17191772), pero los trabajos de otro estudioso de la historia de la estadística, Victor John, de la Universidad de Berna, en 1884, muestran que la tradición «cualitativan se remonta también al siglo XVII, con Herman Conrig (1606-1682), profesor de la Universidad Brunswick de Helmstaedt.

Conrig comenzó en 1660 (o sea, señala Lazarsfeld, casi exactamente en el mismo momento en que Graunt publicaba su trabajo ya mencionado) una serie de conferencias con el tírulo "Noticia Rerum Publicarum», o sea "Información sobre las Cuestiones Públicas».

Lazarsfeld subraya el paralelismo de la preocupación ¿médica? de Petty, que ya hemos visto, con la equivalente de Conrig, quien decía:

Asi como es imposible para un médica dar un consejo para recobrar o preservar la salud si no tiene un buen conocimiento del cuerpo, así también es imposible, para quien no tiene un conocimiento y una conciencia de los hechos de la vida pública, curarla, sea en su totalidad o en cualquiera de sus partes (citado por Lazarsfeld, 1970, p.89).

¿A qué pudo deberse esta diferencia de enfoques? Lazarsfeld apunta una hipótesis muy interesante: para un inglés, ciudadano del imperio más grande de la época, parece lógico adoptar un enfoque cuantitativo y extensivo para el estudio de un "cuerpo social" enorme, que no es posible describir en detalle; para un ciudadano de uno de los 300 minúsculos estados en los que estaban fraccionados los restos del Sacro Imperio Romano Germánico hasta antes de su unificación en la segunda mitad del siglo XIX con Bismarck, parece igualmente lógico un acercamiento cualitativo e intensivo a las ucuestiones públicas" (1970, p.87).

Seguramente hubo muchos otros factores que influyeron en las diferencias que estamos analizando; subrayemos simplemente el ambiente intelectual tan distinto: en Inglaterra la tradición aristotélica no era ya muy fuerte, como lo muestra el éxito de los cuestionamientos de Bacon desde la tercera década del siglo XVIi; en los estados alemanes la tradición pesaba tanto que todavia se escribia en latín.

De hecho el esquema que Conrig propuso para organizar la información "estadística» sigue estrictamente la clasificación aristotélica de las causas: había que reunir información sobre la población y los recursos de un Estado, ciertamente, puesto que ello constituye su "causa material»; pero no sólo sobre eso, sino también sobre los objetivos del Estado en tanto que cuerpo político 
(causa final), sobre sus leyes (causa formal) y sobre sus gobernantes y dirigentes (causa eficiente).

Tres siglos antes de la actual "guerra de los paradigmas", pues, ya se enfrentaban cuantitativistas y cualitativistas.

Parece interesante señalar que los primeros eran ya anglosajones y los segundos germánicos $\mathrm{y}$, además, hay que decir que este primer episodio del enfrentamiento se resolvió con una victoria de los partidarios de la saritmética políticas no sólo en la Gran Bretaña, sino también en los estados alemanes y, en general, en los medios intelectuales de la época: a finales del siglo XVIII los sucesores de Achenwall en Gotinga comenzaron a utilizar matrices, tablas con columnas y renglones, para registrar datos comparativos internacionalmente. Los "cualitativistas" criticaron lo que ellos llamaban despectivamente "estadística de tablas", y el enfrentamiento alcanzó tonos violentos.

Lazarsfeld toma del historiador Victor John algunos ejemplos que muestran la virulencia de la polémica a principios del siglo XIX.

Los partidarios del enfoque cualitativo oponían su propia "estadística", "sutil y distinguida", a la "estadística vulgar" de los cuantitativistas que reducían un gran arte a una "estúpida tarea manual». Según los cualitativistas:

Esos pobres imbéciles difunden la insensata idea de que se puede comprender la potencia de un Fstado conociendo simplemente su supetficie, sa población, su producción y el número de cabezas de ganado que pacen en sus campos. Las maquinaciones a las que se dedican esos criminales uestadísricos políticos" en sus esfuerzos por expresar todo mediante cifras... son despreciables y ridiculas más allá de roda expresión (citado por Lazarsfeld, 1970, p.99).

El prestigio intelectual del pensamiento alemán de finales del xVIIl y principios del XIX (pensemos en Kant, Fichte y Hegel) daba lustre al enfoque "cualitativo". Sin embargo, el enfoque inglés se impuso y hoy, mientras que la tradición iniciada por Conrig ha quedado en el olvido, la que comenzaron Graunt y Petty sigue siendo considerada uno de los fundamentos de la estadística —en su sentido aplicado- y de la demografia modernas.

\section{EL SIGLO XIX: QUETELET YS. LE PLAY. POSITIVISTAS Y EMPIRISTAS VS. HERMENEUTICOS E HISTORICISTAS}

Durante el siglo XIX la historia de la confrontación entre cuantitativisfas y cualitativistas conoció nuevos episodios. Pero antes de relatarlos veamos cómo liegaron a culminar, a principios de esa centuria, los trabajos matemáticos que constituyen la teoría de las probabilidades. 
Estos trabajos, que se remontan al siglo xvr, habían sido totalmente ignorados por los iniciadores ingleses y alemanes de la estadística aplicada a los que nos hemos referido en el apartado anterior. No fue hasta el siglo XIX cuando probabilidad y estadística comenzaron a relacionarse.

Aunque los «juegos de azar" habían sido utilizados desde la antiguiedad, en el siglo XVI comenzó a sistematizarse el conocimiento de las probabilidades. La obra Liber de Ludo Aleae, del gran matemático italiano Gerolamo Cardano (1501-1576), que era también un jugador empedernido, y que fue publicada hacia 1550, comenzó a abrir esa importante avenida de la ciencia moderna que es la teoría de las probabilidades.

A finales del mismo siglo Xvi Galileo (1564-1642) calculó las probabilidades de obtener determinadas puntuaciones con los dados, utilizando procedimientos completamente similares a los que usan en la actualidad. Otro tanto hizo Pascal (1623-1662) en 1654, en una parte de su De Alea Geometriae, enriqueciendo sus propias opiniones con las de Pierre de Fermat (1601-1665) con quien intercambió ideas por carta ese año. Christian Huyghens (1629-1695), matemático holandés que visitó a Pascal en París en 1655, se benefició de las ideas de ambos, las desarrolló, y las publicó en 1657, en su De Ratriociniis in Ludo Aleae, en donde por primera vez se presenta el concepto abstracto de esperanza matemática.

El primer gran matemático de la familia Bernouilli de Basel, Jakob I (16541705), produjo en forma póstuma la primera gran obra teórica: Ars Conjectandi (1713), en la que se incluáa una teoría de las combinaciones y las permutaciones.

Por la misma época, un hugonote francés exiliado en Inglaterra (donde se relacionó con Newton y Halley), Abraham de Moivre (1667-1754), hacía avanzar el tema con sus obras De Mensura Sortis (1711), The Doctrine of Chances (1718) y Miscellanea Analytica (1730); en 1733 derivó la curva normal como una aproximación de la ley binomial.

En 1763 apareció en forma póstuma el Essay Towards Solving a Problem in the Doctrine of Chances, de Thomas Bayes (1702-1761), que dio inicio a la estadística inductiva.

Y es al comenzar el siglo xiX, en 1812, cuando aparece la obra clásica de la disciplina: la Théorie Analythique des Probabilités de Laplace (1749-1827). Escrita para presentar los métodos matemáticos desarrollados por su autor para predecir la probabilidad de los fenómenos naturales en el campo de la astronomía y de la física, se vio complementada en 1814, al publicarse la segunda edición, por una obra de divulgación, el Essai Philosophique sur les Probabilités, en el que el nuevo marqués aplicaba sus teorías a problemas cotidianos de carácter fortuito y a las estadísticas vitales.

Por $\mathrm{fin}$, unos años más tarde, encontramos al último antecedente de nuestra historia: el "príncipe de los matemáticos" del siglo xIX, Carl Friedirch Gauss (1777-1855). 
Una de sus muchas contribuciones fue el desarrollo de la curva normal, derivada teóricamente por De Moivre casi cien años antes, y que utilizada por Carl Friedrich llegó a ser la más famosa de las distribuciones de probabilidad: la campana de Gauss, que representaba la distribución de los errores que se daban en las mediciones geodésicas en las que se ocupó nuestro autor a principios de la década de 1820 .

La relación encre, por una parte, los desarrollos teóricos sobre la probabilidad, de Cardano a Laplace y Gauss y, por otra, la "estadística» empírica de Graunt y William Petty a Suessmilch fue la obra, antes que nadie, de Lambert Adolphe Jacques Quételet (1796-1874), en quien se encuentra "la primera tentativa de fundar una ciencia social cuantitativas (Boudon, 1970, p. 38).

Nacido en la ciudad de Gante, Quételet se dedicó primero a la pintura y la literatura, pero luego estudió matemáticas doctorándose en su ciudad natal en 1819.

Enviado a París en 1823 como preparación para crear un observatorio astronómico en Bruselas, entró en contacto con los matemáticos de la Ecole Polytechnique y, especialmente, con Laplace, con quien se introdujo a la teoría de las probabilidades.

En 1826 ayudó a la Comisión Real de Estadística a preparar un censo de la población belga y preparó unas tablas de mortalidad a partir de los datos obtenidos. En 1827 comenzó sus famosos análisis de las estadísticas de criminalidad. En 1831 publicó un importante estudio sobre la estatura de las personas, y otro sobre la inclinación al crimen a diversas edades. En 1832 apareció un tercer trabajo sobre el peso de la gente. Integrando estos trabajos, en 1835 apareció su obra Sur l'Homme et le Dévelopement de ses Facultés. Physique Sociale (Lazarsfeld, 1970, p.104-105).

Hasta entonces Quételet se limitaba a calcular los promedios y las tasas de ciertos aspectos (hoy diríamos "variables") como la talla, el peso o el número de delitos cometidos en relación con la edad u otra característica demográfica. Pero a partir de 1840 comenzó a interesarse por la distribución de las variables, al observar que la representación gráfica de la talla y el peso de las personas se parecía mucho a la de la distribución de los errores de medición, que Laplace y Gauss habian estudiado. Desde entonces se convenció de que la distribución de las propiedades físicas de la gente puede asimilarse a una distribución binomial o normal (Lazarsfeld, 1970, p.105-106).

El carácter sorprendentemente estable de tales distribuciones en diferentes páses y épocas lo llevó a la idea de que constituyen uleyes» sociales.

Como es fácil ver comparando las "variables" talla y peso con la de inclinación y propensión (penchant) al crimen Quétclet no se limitó a estudiar la distribución de propiedades "físicas", sino que también dedicó su atención a propiedades "morales", lo que le hace ser también un precursor de los trabajos 
de medición en ciencias del hombre. Lazarsfeld observa que hay que esperar casi un siglo, hasta el articulo de L.L.Thurstone de 1927-28, "Attitudes can be mesured", para encontrar algo parecido a los esfuerzos de Quételet por medir las inclinaciones, "Les penchants et leur mesure", (1970, p.125).

Aunque lo postuló repetidamente, Quételet de hecho no realizó nunca lo que hoy Ilamaríamos "trabajo de campo" para obtener "datos" sobre las "propiedades" que le interesaban; él siempre utilizó "estadísticas" disponibles, de las muchas que se recopilaban ya en su época sobre los más diversos aspectos, siguiendo los impulsos iniciados por los autores que hemos visto antes.

El que desarrollaría ese otro aspecto de la investigación, y precisamente con el énfasis puesto en el otro estilo que hoy llamariamos cualitativo, fue un ingeniero francés, Pierre Guillaume Frédéric Le Play (1806-1882).

Formado en I'Ecole Polytechnique, Le Play se vio fuertemente influenciado por los períodos revolucionarios de la historia de Francia que vivió, concretamente las revoluciones de 1830 y 1848 , y la Comuna de París de 1870.

Le Play realizó numerosos trabajos de observación detallada de familias obreras, publicadas en forma de monografias, que lienan extensos volúmenes de brillante estilo.

Considerado por ello pionero de la investigación empírica, Le Play presenta una situación paradófica en cuanto se refiere a su influencia: admirado ciegamente por un grupo reducido de fervientes seguidores, y a la vez casi ignorado fuera de su pequeño círculo. Ambas cosas parecen explicarse por la personalidad carismática de nuestro personaje así como por la contundencia de su peculiar ideología, en la que se mezclaban convicciones teológicas (consideraba que la salvación de la sociedad europea radicaba en que se volviera al respeto de los diez mandamientos y de la autoridad paterna) con una honda preocupación por la situación de los trabajadores pobres, vagas aspiraciones de cariz socialista y un rechazo de ciertos pensadores o lugares a los que atribuía un carácter pernicioso, como Adam Smith, Rousseau, París y los Estados Unidos.

A diferencia de otros pioneros del trabajo empírico como el inglés Charles Booth (1840-1916), quien permanentemente buscó afinar sus procedimientos, incorporó técnicas cuantitativas y supeditó siempre sus planteamientos teóricos a sus hallazgos empíricos. Le Play no mostró avances en su metodología a lo largo de cincuenta años, rechazó violentamente la estadística, y subordinó la información empírica a sus tajantes planteamientos ideológicos, si bien lo penetrante de sus observaciones, la audacia de sus generalizaciones, la abundancia de hechos a que se refiere, la viveza de su estilo y la claridad de su redacción hacen cautivante su lectura (Lazarsfeld, 1970, p.146-151).

Lazarsfeld afirma que la historia de la escuela de Le Play después de la muerte del maestro "es un curioso ejemplo de lo que puede pasar con una tra- 
"Papersn: Revista de Sociologia

dición enfocada a la investigación cuando se transforma en sectan, y resume la cuestión de esta forma:

Estamos en presencia de una corriente de pensamiento creada por una personalidad carismática, que aporta una innovación capital en ta metodología de las ciencias sociales, al mismo tiempo que mezcla estrechamente inquebrantables conviciones de orden ideológico y práctico. Algunos de sus discípulos se sienten divididos entre dichas convicciones, que ellos adoptan, y los progresos que quieren desarrollar en la metodología. En una situación normal, todo sabio que sea un precursor se gana el respeto de sus discípulos, pero al mismo tiempo se admite sin problema que ćstos deberán ir más allá de lo que haya descubierto su maestro. En un contexto carismácico, por el contrario, se observa un conflicto entte la fidefidad a la secta y el progreso de la ciencia. Este conflicto sería de poco interés para nosotros si no constatáramos la rapidez con la que los seguidores de Le Play perdieron el lugar que ocupaban en la sociologia francesa (1970, p.142-143).

Los trabajos cuantitativos y cualitativos de Quételet y Le play se desarrollaron paralelamente sin enriquecerse ni contraponerse mutuamente, sino más bien ignorándose, si bien Le Play, como ya hemos dicho, se oponía drásticamente al uso de la estadística (según Bureau, citado por Lazarsfeld, 1970, p.153).

Fue en otro contexto en el que se dio otro episodio del enfrentamiento entre los dos estilos de investigación a los que nos hemos venido refiriendo: en un bando podemos colocar a los partidarios de la investigación según el modelo de las ciencias de la naturaleza, entre los que podemos situar, con diferentes matices, a Auguste Comte y John Stuart Mill.

El primero (1798-1857), fundador del positivismo y creador de la palabra "sociología", aspiraba a que dicha disciplina llegara a ser "la reina de las ciencias", siguiendo el modelo de la física, si bien él no planteó expresamente la tesis del monismo metodológico, que pertenece a los neopositivistas del Círculo de Viena, en los años veinte del siguiente siglo.

El segundo, Mill (1806-1873), con una influencia real sobre la investigación mucho mayor, como precursor inmediato del diseño experimental, y en quien algunos ven al pensador que estableció, desde 1843, la agenda de la investigación social para más de un siglo (Costner, 1988, p.42).

En el otro bando, deberemos colocar a un grupo de pensadores que consideraron que el estudio de las cuestiones sociales (o "espirituales", o "morales») tendría que abordarse de una manera radicalmente distinta de la de las ciencias de la naturaleza.

Varios pensadores alemanes plantearon la distinción entre ciencias de la naturaleza y del espíritu (Naturwissenschaften vs. Geisteswissenschaften), así como la oposición correlativa entre explicación y comprensión (Erklärungvs. Verstehen). 
Sin hablar de Hegel (1770-1831), cuya filosofia de la historia tiene afinidades evidentes con estas corrientes, otros pensadores alemanes se encuentran en el origen de la hermenéutica moderna: Schleiermacher (1768-1834), von Ranke (1795-1886) y Droysen (1808-1884), ésre último el primero en utilizat la distinción entre explicación causal vs. comprehensión de la actividad intencional (Coreth, 1972, p.31-35).

Wilhelm Dilthey (1833-1911), quien fundamentó la comprensión en la identidad sujeto-objeto, propia, en su opinión, de las ciencias del espíritu.

Wilhem Windelband (1848-1915), quien subrayó más bien que las ciencias de la naturaleza tratan de encontrar leyes generales mientras que, desde su punto de vista, las del espíritu estudiarian hechos únicos e irrepetibles, introduciendo la distinción entre disciplinas nomotéticas e ideográficas.

Y por fin Max Weber (1864-1920), quien, siguiendo a Rickert (18631936), destacó la importancia de la comprensión como método de las ciencias del espíritu, en las que los objetos están dotados de valor (Mardones y Ursúa, 1982, p. 22-23).

Interrumpido por la Primera Guerra Mundial, de 1914-1918, este enfrentamiento resurgiría después, como veremos enseguida.

\section{DE 1890 A 1939: ESTADÍSTICA VS. ESTUDIOS DE CASO}

El tercer episodio de la confrontación que estamos estudiando, ya muy cercano a nosotros y sin embargo no muy conocido en nuestro medio, tiene lugar de finales de siglo pasado hasta bien entrado el nuestro, fundamentalmente en los Estados Unidos.

Los preámbulos de esta fase los encontramos en el importantísimo desarrollo de la estadística moderna a partir de la última década del siglo XIX.

En su artículo sobre la "Historia del Método Estadístico", escrito para la Enciclopedia Internacional de Ciencias Sociales, Sir Maurice G. Kendall escribe:

Si tenemos que elegir una fecha como origen de la moderna reoría de la estadística podemos señalarla, algo arbitrariamente, en 1890 . Francis Galton tenla entonces sesenta y ocho años, pero todavía tenía por delante veinte años de vida productiva. Un profesor de economía, Francis Ysidro Edgeworth (entonces de cuarenta y cinco años), estaba llamando la atención sobre las regularidades estadísticas en los resultados de las elecciones, en los versos griegos y en el aparejamiento de las abejas, e iba a proponer una notable generalización de la ley del error. Un joven llamado Pearson (de treinta y cinco años) se acababa de unir al biólogo Waiter Weldon del University College de Londres y estaba meditando las lecciones que finalmente se convertirian en The Gram- 
"Papers": Revista de Sociologia

mar of Science. Un estudiante llamado Georgc Udny Yule, a los veinte años de edad, había atraido la atención de Pearson. Yen aquel año nació el más grande de todos ellos: Ronald Aylmer Fisher... (1974, p. 406-407).

Kendall señala que, sin menospreciar las aportaciones de otros países, el desarrollo de la estadística en la época que nos ocupa fue obra primordialmente de autores ingleses, e indica cómo en 1890 Pearson, estimulado por Galton, estaba comenzando a estudiar las distribuciones bivariantes, lo que llevó a las medidas de asociación y contingencia para lo que entonces se llamaban atributos, y a la cotrelación y la regresión para las llamadas variables (1974, p. 407).

En 1900 Pearson redescubrió la distribución que ya había encontrado el alemán Helmert; en 1908 su alumno William Gosset elaboró la distribución $t$ que bautizó con el seudónimo de Student.

De 1913 a la época de la Segunda Guerra Mundial, Fisher desarrolla el diseño experimental moderno, pasando del experimento determinístico de $\mathrm{Ba}$ con y Mill al estocástico.

Entre 1925 y 1935 nacen la teoría de la estimación de Fisher y la teoría del contraste de hipótesis, con Jerzy Neyman y Egon Pearson, hijo éste último de K. Pearson (Kendail, 1974, p. 408).

Como ha podido apreciarse, nos hemos referido hasta ahora a los desarrollos de la estadística teórica, que fueron extraordinarios en el medio siglo al que nos referimos.

En cambio, la utilización de esas herramientas en la investigación empírica fue mucho más lenta y desigual.

Ch. Booth y S. Rowntree comenzaron a realizar encuestas sociales en Inglaterra a finales del siglo pasado, y A.L. Bowley continuó con esa tarea en las primeras décadas del nuestro, aplicando la teoría estadística.

En el campo de la psicología se avanzó bastante en lo relativo a la medición, con la construcción de pruebas de inteligencia desde principios del siglo, tanto en Francia con Binet como, sobre todo, en los Estados Unidos con Thorndike y Thurstone.

En 1940 Thorndike publicó su importantísima obra An Introduction to the Theory of Mental and Social Measurements, en la que aparece su cellebre afirmación «todo lo que existe, existe en cierta cantidad y por lo tanto puede medirse".

En la sociología europea de principios de siglo, fue Weber quien, aunque primordialmente interesado en las investigaciones históricas que to hicieron famoso, en varias ocasiones se ocupó cambién de encuestas empíricas. Durkheim, por su parte, hizo ingeniosos análisis que anticipaban los enfoques multivariados posteriores. No obstante, ninguno concentró su atención en las técnicas cuantitativas, ni pareció haber tenido conciencia del potencial que representaban los avances de la estadística matemática. 
A diferencia de Europa, y por circunstancias de orra índole, es en Estados Unidos donde la investigación social se desarrolló con mayor fuerza durante la primera mitad del siglo XX. En el campo de la sociología fue el lugar privilegiado de tales desarrollos la Universidad de Chicago. Establecido en 1892, y dirigido desde entonces hasta 1924 por Albion Small, el departamento de Sociología de Chicago destacó rápidamente como centro de la nueva investigación social.

En especial, la aportación de William I. Thomas, el cuarto estudiante que recibió el doctorado en Sociología por Chicago, incorporándose de inmediato al personal académico, significó el desarrollo de importantes trabajos empíricos de corte cualitativo que culminaron con la publicación -entre 1918 y 1920 - de los tomos de su famosa obra (con Florian Znaniecki) The Polish Peasant in Europe and America (Bulmer, 1986, p. 35-37).

La llegada de Robert Park al departamento -en 1913, por invitación de Thomas -, seguida por la de Ernest Burgess en 1916, marcó el inicio de un período de auge para la sociología de Chicago.

De 1915 en adelante, mientras Chicago se consolidaba, Columbia se debilitaba, por lo que la preeminencia de la primera se hizo más clara. Así, de . los primeros trece presidentes que tuvo la American Sociological Sociery desde su fundación en 1905 hasta 1923, sólo tres estaban relacionados con Chicago. En cambio dieciséis de veintisiete presidentes de la ASS de 1924 a 1950 fueron profesores o egresados de Chicago, mientras que sólo siete lo fueron de Columbia.

Y entre 1912 y 1933 Chicago produjo ocho presidentes de la ASS y Columbia ninguno (Bulmer, 1986, p. 38-44).

Bajo la influencia de Thomas, así como la de los psicólogos Georges $\mathrm{H}$. Mead y Charles $\mathrm{H}$. Cooley, y gracias a los trabajos de sociología urbana de Park y Burgess, la escuela sociológica de Chicago promovió el desarrollo de técricas cualitativas de investigación social, en particular los estudios de caso. El surgimiento de una nueva corriente que, con Herbert Blumer, llegaría a conocerse más tarde como interaccionismo simbólico, es un indicador suficientemente claro de la importancia de este enfoque en aqueilla época en Chicago.

Mientras tanto, el enfoque cuantitativo no se desechaba, pero era cada vez mas débil, hasta que, al jubilarse Small en 1926, se consideró conveniente reforzarlo, y se atrajo al departamento a un destacado metodólogo que estaba descontento por el declinar de Columbia, William F. Ogburn, quien aceptó trasladarse a Chicago en enero de 1927 (Bulmer, 1986, p.170).

Para 1929 Chicago estaba en condiciones de inaugurar un nuevo edificio para albergar un Instituto de Investigaciones Sociales.

Para entonces también Ogburn se había puesto en contacto con otros destacados investigadores de Chicago, partidarios del enfoque cuantitativo: James 
A. Field, profesor de estadística, alumno de Pearson en Londres; L.L. Thurstone del departamento de Psicología, uno de los pioneros en el campo de la medición; Henry Schultz, profesor de Economía, que también había estudiado en Londres con Pearson y Bowley; y Charles Merriam, Harold Gosnell y Helbert Simon de Ciencias Políticas, además del biólogo Sewall Wright, creador del «Path Analysis".

Entre los alumnos que en 1929 preparaban el doctorado en Sociología con Ogburn encontramos a Philip Hauser y a Samuel Stouffer. Este último, después de doctorarse, pasó los años de 1931 y 1932 en Londres, estudiando con Pearson, Yule, Fisher y Bowley, antes de reincorporarse como profesor del departamento de Sociología de Chicago en 1935; luego continuaría su brillante carrera en Harvard.

Una idea de lo avanzado que resultaba para la época el trabajo de Ogburn desde un punto de vista metodológico, nos lo da el hecho de que, al analizar en 1929 los resultados de las elecciones presidenciales del años anterior, utilizó ya correlaciones parciales para controlar estadísticamente los efectos de interacción que pudieran darse entre las variables (Bulmer, 1986, p.172-181).

La coexistencia de los partidarios del enfoque cuantitativo y los del cualitativo, que al principio era pacífica gracias en parte a la influencia aglutinadora de Burgess, se fue haciendo paulatinamente más difícil.

Ogburn logró que en el frente del nutevo edificio de Investigación en Ciencias Sociales, inaugurado, como hemos dicho, en 1929, en litgar de la famosa frase de Aristóteles "anthropos zoon politikon" se inscribiera esta otra, extractada de una conferencia pronunciada en 1883 por el destacado físico inglés Lord Kelvin: "cuando no podemos medir, nuestro conocimiento es pobre e insatisfactorion.

Nos han llegado las versiones de la forma en que reaccionaron otros profesores al ver tal inscripción. Un oponente de Ogburn, Jacob Viner, comentó: "Y cuando podemos medir, nuestro conocimiento sigue siendo pobre e insatisfactorio"; por su parte Frank Knight, rival de Thurstone, dijo: "Y si algo no se puede medir, tú mídelo de todos modos" (Bulmer, 1986, p. 212).

Ogburn defendía un acercamiento cuantitativo y riguroso que desconfraba de la teorización y de la posible distorsión provocada por valores y actitudes, por lo que también consideraba negativo el compromiso político e ideológico. Se dice que, cuando se publicó el famoso estudio de caso de Robert Lynd titulado Middletown, Ogburn afirmó que era "demasiado interesante para ser cientificon (Bulmer, 1986 p.182).

Defendiendo el acercamiento cualitativo de los estudios de caso, Cooley decía a su vez que las mediciones eran sólo una forma de ser preciso, y que había otras como por ejemplo una película animada; y afirmaba que lo que se necesitaba en la investigación social era la habilidad de registrar "totalidades vivien- 
tes", "la percepción, registro y comprensión incegrales de acciones fundamentales". Según Cooley, el objetivo del sociólogo debe ser hacernos ver la vida humana como una actividad dramática, $y$ uparticipar en los procesos mentales que forman parte de la función humana y son accesibles a la observación simpatizante con la ayuda de los gestos y el lenguaje" (Bulmer, 1986, p.183).

Como ya se dijo, Burgess tenía una posición conciliadora. En un artículo publicado en 1927, y titulado precisamente "Statistics and Case Srudies as Methods of Sociological Research", escribía:

Los métodos de la estadística y los estudios de caso no se oponen entre sí, sino que se complementan mutuamente. Las comparaciones y correlaciones estadísticas pueden con frecuencia sugerir puntos a investigar mediante estudios de caso, y los materiales documentales, al revelar procesos sociales, inevitablemente abrirán el camino a índices estadísticos más adecuados. Рara que las estadísticas y los estudios de caso puedan representar una aportación plena como herramientas de la investigación sociológica tiene que dársetcs igual teconocimiento a ambos, as como amplias oportunidades para perfeccionar sus propias técnicas. Al mismo tiempo, es seguro que la interacción de los dos métodos será fructífera, (citado en Bulmer, 1986, p. 185).

El mismo Stouffer, cuya tesis había mostrado la superioridad de procedimientos formales de medición tipo Thurstone en relación con acercamientos cualitativos de tipo historias de vida, para estudiar un tema dado distaba mucho de despreciar los estudios de caso. Opinando sobre algunos trabajos de ese tipo en el terreno de la criminología, Stouffer escribia en 1934:

Es dificil imaginar una ecuación matemática, por complicada que sea, que tenga mucho valor para estudiar la delincuencia si no va acompañada por datos no cuancizativos incluyendo, tal vez, documentos cuya interprctación es esencialmente un procedimiento artístico... El trabajo The Jack Roller puede estar más cerca del arte que de la ciencia, cal como se usa convencionalmente esca palabra por los científicos, pero si nos ayuda a comprender la delincuencia por algún tiempo tiene que ser sociología, y de la buena... Limitarnos a medir esterilizaria la investigación... Deberíamos sentirnos libres para urilizar las récnicas que queramos sin tener que perder el tiempo preguntándonos sobre un trabajo concreto, en forma inúrilmente escolásrica: ¿es ciencia o no? (citado en Bulmer, 1986, p.186).

Pero la polémica se agudizó al pasar el tiempo. Philip Hauser recuerda que, en los días de campo que hacían los profesores del departamento, sc organizaban juegos de softball que oponían a estadísticos y partidarios de los estudios de caso. Esto sucedía todavía al comenzar los años treinta. A mediados y a finales de esa década los adversarios ya no querían jugar juntos, ni siquiera en equipos diferentes. En 1935 y 1936 la American Sociological Society se divi- 
"Papers": Revista de Sociologia

dió y se alejó de Chicago, creando su propia revista, la American Sociological Review. Chicago siguió publicando el American Journal of Sociology, y bajo su influencia surgió la Sociological Research Association.

Interrumpida seguramente por la guerra, la confrontación conocería nuevas etapas después.

\section{DE 1940 A 1989: NEOPOSITIVISMO Y POSTPOSITIVISMO VS. TEORIA CRITICA Y HERMENÉUTICA}

Además de interrumpir momentáneamente la polémica, la Segunda Guerra Mundial constituyó la ocasión de nuevos e importantes avances en el campo de la metodología cuantitativa.

En efecto, las grandes investigaciones patrocinadas por el ejército que se plasmaron después en la famosa obra The American Soldier, permitieron trabajar juntos al mejor discípulo de Ogburn, Samuel Stouffer --quien, como hemos visto, pasó un par de ańos estudiando con los grandes estadísticos ingleses - y a Paul Felix Lazarsfeld, quien había emigrado de Austria en 1933 y que, sin conocer los desarrollos de Karl Pearson y compañía, aportaba su formación matemática, su experiencia de investigación psico-social (que había producido ya Die Arbeitslosen von Mariental (Los desempleados de Marientall, y muchos trabajos más desde 1920), su indiscutible creatividad, y algunos valiosos colegas como Hans Zeisel y Marie Jahoda.

En 1946 Lazarsfeld presentó, en el congreso de Cleveland de la American Sociological Association, un trabajo sobre "La interpretación de las relaciones estadísticas como procedimien to de investigación", en el que describe por primera vez lo que luego se llegará a conocer como uel modelo de elaboración de Columbia».

En 1950, el mismo año en que publicó - junto con Merton- su trabajo sobre el objetivo y método de The American Soldier, Lazarsfeld elaboró una propuesta - también con Merton- para la creación en Columbia de un instituto dedicado a la formación metodológica para la investigación social.

De este famoso "Proyecto Columbia" se derivaron obras clásicas de metodologia, como The Language of Social Research, del mismo Lazarsfeld con M. Rosenberg (1955), o Survey Design and Analysis, de Herbert Hyman (1955). Allí se formaron no pocos de los más importantes «metodólogos» cuantitativos que aún están en activo.

Pero el impulso que representó el modelo de Columbia para la adopción del análisis multivariado en sociología no fue sino uno entre numerosos avances realizados en la época: de los años cincuenta datan también la aplicación del análisis factorial y del análisis de correlación canónico; el desarrollo de los 
trabajos de Yule sobre series temporales y autorregresión, así como de los procesos de tipo estocástico estudiados por Andrew Markov y otros; por entonces se pusieron también de moda los métodos de distribución libre, o no paramétricos, así como las técnicas de probabilidad inductiva de tipo bayesiano; en el campo de la estadística inferencial se desarrolló la teoría del muestreo secuencial, así como la teoría de las funciones de decisión para la aceptación o el rechazo de hipótesis (Kendall, 1974, p. 409-410).

Por lo que se refiere a la medición, en 1940 apareció el informe en el que un comite de la British Association for the Advancement of Science concluía que las técricas utilizadas en las ciencias del hombre no satisfacían plenamente las condiciones establecidas en 1917 por Campbell para la medición física; a partir de entonces dichas técnicas se desarrollaron y perfeccionaron con los trabajos teóricos de Gulliksen en 1946 y Stevens en 195I, así como con los avances prácticos de Guttman en los años cuarenta, Rasch en los cincuenta, etc. (cf. Keats, 1988).

Después de 1960 ha habido desarrollos tan importantes como la aplicación del Path Analysis en la investigación social (con H. Blalock, R. Boudon y Otis D. Duncan), el análisis loglinear, el escalamiento multidimensional, el análisis exploratorio de datos (DEA) y el meta-análisis, así como las teorías de generalizabilidad y de respuesta al ítem en el campo de la medición (Keeves, 1988).

Seguramente puede afirmarse que, de la Segunda Guerra a finales de los años cincuenta, el predominio de la investigación de corte cuantitativo fue abrumador. Pero ya a principios de la siguiente década, dicho predominio se vio fuertemente cuestionado. La "guerra de los paradigmas" no comenzó en los años ochenta, sino dos décadas antes.

La famosa polémica entre Sir Karl Popper -el principal heredero crítico del neopositivismo del Círculo de Viena - y Theodor Adorno --el principal representante en ese momento de la Escuela de Frankfurt- tuvo lugar en Tubinga, en un congreso de la Asociación Alemana de Sociología, en 1961. Postpositivismo y teoría crítica comenzaban una polémica que no ha terminado treinta años después.

De la segunda mitad de la década de los cincuenta datan también, por una parte, el resurgimiento de la hermenéutica y, por otra, el del neomarxismo, cuyos planteamientos filosóficos se manifestaron en el ámbito de las ciencias del hombre. Emparentados con la hermenéutica se desarrollaron el interaccionismo simbólico, la etnometodología y la sociología fenomenológica, mientras que a la filosofía de inspiración dialéctica correspondieron numerosos trabajos neomarxistas.

La importancia de estos enfoques desde el punto de vista metodológico, ya durante los años sesenta, puede apreciarse fijándose en la fecha de la primera publicación de obras como las siguierates: 
"Papers": Revista de Sociologia

Method and Measurement in Sociology, de A.V. Cicourel, 1964.

The Human Perspective in Sociology, de Severyn T. Bruyn, 1966

The Social Construction of Reality, de P. Berger y Th. Luckmann, 1966.

The Discovery of Grounded Theory, de Glaser y Strauss, 1967

Studies in Ethnometodology, de H. Garfinkel, 1967.

The Phenomenology of the Social World, de A. Schutz, 1967.

Le Metier de Sociologue, de Bourdieu, Passeron y Chamboredon, 1968.

De la misma época, aunque las publicaciones relacionadas son posteriores, data el surgimiento de la investigación acción participativa, investigación militante, o equivalentes en América Latina.

La fuerza de las críticas a los enfoques convencionales de la investigación llegó a ser tan fuerte a finales de los años sesenta en algunos lugares, como Alemania, que el autor de tres importantes libros sobre filosofia de la ciencia aparecidos entre 1969 y 1971 escribió, analizando la impermeabilidad de los críticos marxistas frente a cualquier otra crítica o argumento que se les opusiera:

El marxismo cn su forma actual sin duda es arractivo y fácilmente accesible para los jóvenes. Pero quien ha cruzado una vez las barreras inferiores hacia la doctrina marxista ya no encuentra una salida que lo saque de allí, pues aquella le ofrece un sistema doctrinal que lo inmuniza plenamente contra la crítica de fuera y penaliza de antemano su voluntad de apearse de elia... En principio es posible, y lo seguirá siendo, someter a discusion, con argumentos, el marxismo... Si no tienen lugar tales discusiones... la razón está en que el clima socioespiritual que se desarrolla y se hace cada vez más intenso entre los jóvenes no tolera tales discusiones... quien ha llegado a conocer la propensión de los hombres hacia las tendencias dominantes, su impulso a la uacomodación ávida a la modan (Adorno), se entregará a pocas ilusiones en este asunto. Los problemas como tales permanecerán, pero serán elaborados y se intentará resolverlos solamente en el sentido del marxismo. Solo muy pocos espíritus independientes se atreverán a manifestar una critica serena, pero no setán entendidos. Quizá no queda sino depositar esea crítica en archivos y dejarla invernar, hasta que, con nuevos cambios históricos, nazca una nueva inteligencia para argumentaciones proscritas hoy como burguesas (Seiffert, 1977, p. 487-488).

En 1991 el clima intelectual - por lo menos en gran parte de Europa--es muy diferente al de 1971, habiéndose producido ya "cambios históricos"; pero - como sabemos bien quienes conocemos el desfase cronológico con el que llegan las nuevas corrientes de pensamiento a los países "periféricos"- la virulencia de los enfrentamientos varia mucho según los lugares y, también, según las disciplinas. Así podemos encontrar un desfase entre la polémica en el campo de la investigación educativa y la del campo sociológico; y podemos ver como reaparece la oposición "ciencia burguesa-ciencia proletaria" de la glaciación estaliniana en ciertas oposiciones recientes, como la de la ciencia femenina vs. 
masculina o ciencia negra vs. blanca, como resurge también el rechazo de la lógica formal que hacía el marxismo más ortodoxo en nombre de la dialéctica en los años cincuenta. En 1978, por ejemplo, Mittroff y Kilman escribían:

La ciencia convencional es marcadamente masculina en su orientación, reflejando los tradicionales valores machistas estereorípicos, a saber: es de "nariz duram, objetiva, libre de valores; rechaza lo ambiguo, lo especulativo, lo vago, lo hermoso y lo bueno. Por el contrario, una ciencia femenina no teme a lo bueno, lo especulativo, lo vago o lo único; al revés, corteja abiertamente con ellos, los confronta francamente y hace de ellos virtudes positivas (citados en Reason y Rowan, 1981, p. 51).

Estos dos autores dicen que uno de los problemas más difíciles que hay que superar para hacer ciencia en forma alternativa es la limitación que constituye la lógica aristotélica, con sus principios de no contradicción y de tercero excluido; pero dicen que, si no logramos superar tal obstáculo y tratamos de investigar alternativamente pero siguiendo la lógica tradicional, haremos mala investigación convencional en lugar de investigación alternativa.

\footnotetext{
Necesitamos aprender, porque la lógica tradicional y los niveles ordinarios de conciencia no son apropiados cuando trazamos de investigar según el nutevo paradigma (1981, p. 114).
}

Según estos autores hay que reconciliar e integrar elementos rivales, como "el hemisferio cerebral derecho y el izquierdo, lo masculino y lo femenino, el yin y el yang, el entendimiento y las emociones, la sensación y la intuición’ (p.117).

$\mathrm{Y}$ más adelante nos dicen que, para lograr ese nivel superior de conciencia, lo peor que se puede hacer es concentrarse en ello, ya que resulta contraproducente, y que la "manera de tratar de aprender esto es dejando de intentarlo; la manera de concentrarse en ello es no concentrándose, y para atraparlo hay que dejarlo ir. El secreto está en dejar de pensar y mantenerse consciente de la propia experiencia en el permanente fluir del presente» (p. 122).

Por su parte, los partidarios de la investigación-acción participativa, además de exigir la intervención de la comunidad, y el compromiso político de los investigadores, con miras no sólo a incrementar el conocimiento de la realidad sino, más fundamentalmente, a transformarla radicalmente mediante un cambio en las relaciones de poder a favor de los grupos oprimidos, postulan la superioridad epistemológica de su enfoque, por considerar que:

Se trata de un método de investigación más ciencífico que la investigación tradicional, en el sentido de que la participación de la comunidad facilica un análisis más preciso y más auténtico de la realidad social (Int. Council for Adult Education, 1977, citado en Landshecre, 1986, p. 282). 
Otra expresión muy reciente y categórica de este tipo de posturas la encontramos en una comunicación aparecida en el boletín de la American Sociological Association, Footnotes, del mes de noviembre de 1990. Sus autores, criticando la contribución que, en su opinión hace la investigación convencional a la destrucción de la ecología del planeta, concretamente por hacer que se talen muchos árboles para producir las grandes cantidades de papel que requiere el procesamiento y la publicación de numerosos resultados cuantitativos, escriben:

Después de mucha discusión flegamos al acuerdo de que la tesis debcría tener un enfoque cualicarivo. Ningún númcro. Más árboles salvados, más ozono, más "verdad"... No habrá moneones de papel de computadora desperdiciado, ni montones de empolyados cuestionarios respondidos. De hecho, en una versión extrema de lo que gustamos llamar metodologia cualitativa ambiental toda la investigación debería ser integramente oral o visual, plenamente orgánica, y no habría ningún tipo de informe académico (ni sicuiera utilizando papel reciclable); el informe final se escenificaría como una pieza de teatro, una danza o una canción ante la comunidad académica. Ahora bien, ése es precisamenre el tipo de ciencia social del próximo milenio. O, por lo menos, de los próximos años" (Renard y Baker, 1990, p.18).

No hay necesidad de caricaturizar para ver los extremos a los que puede llegarsc en la polémica. Basta citar al pie de la letra, aun a riesgo de consumir un poco más de papel.

Por supuesto, los partidarios de la investigación convencional no son tampoco benignos en sus críticas a los "alternativos". Ya en $1971 \mathrm{E}$. Borgatta escribía, en la introducción al primer volumen de la serie Sociological Methodology, que le parecía detectar dos tendencias inadectadas en el desarrollo de la metodología en los años sesenta: una cra la tendencia a dar el doctorado en sociología a quienes alcanzaran el nivel de un licenciado en matemáticas en esta última disciplina, independientemente de sus conocimientos sociológicos; la otra era un incremento de la "agresión matemática", o sea la tendencia a descalificar al adversario juzgándolo como ignorante y obsoleto si no logra comprender la jerga de las matemáticas (1971, p. x).

Tratando de encontrar un equilibrio que reconozca las limitaciones de ambos enfoques y combine sus puntos fuerres, ottos autores adopran posturas conciliadoras análogas a la que encontramos en Burgess medio siglo atrás. R.C. Rist, por ejemplo, decía ya en 1977:

Evaluación dura contra blanda, cuantificaciones contra descriprores, cicntificos contra críticos, rigor contra intuición... es simplemente una perogrullada decir que las dicotomias expresadas por los estercoripos anteriores, tan gastados ya, han dominado durance demasiado riempo la discusión comparativa de las 
diversas estrategias de investigación en el campo educativo. Se reduce así la complejidad y los marices de diferentes acercamientos a posturas polarizadas simplistas y rígidas... (citado por De Landsheere, 1986, p. 343).

Y es el mismo Rist, especialista en investigación etnográfica, quien llama la atención sobre el carácter "sectarion del "movimiento" que propugna el rechazo de los métodos convencionales, y la adopción exclusiva de los alternativos. En su artículo "Etnografía tipo "guerra relámpago" (Blitzkrieg Ethnography): sobre la transformación de un método en un movimienton (1980) Rist escribe:

La etnografía se está convirtiendo en un manto para legicimar mucho trabajo de mala calidad, pobremente realizado y ral concebido. Cuando se cuestiona tal trabajo la respuesta consiste en utilizar terminología defensiva... El método etnográfico está en boga. Irónicamente, cuanto más in es el mérodo, menos se parece a un instrumento de investigación disponible y más se acerca a las características de un movimiento... (cizado por Gibaja, 1988, p. 84).

Nos encontramos nuevamente con el fenómeno de la transformación en secta (movimiento, en la terminología de Rist) de una corriente cientifica, y su consecuente empobrecimiento, como había sucedido con los seguidores de Le Play hace cien años.

El ingrediente ideológico señalado por Seiffert en relación con el marxismo en 1971 sigue también presente.

\section{BIBLIOGRAFÍA}

Borgatta, E.F. (ed.), Sociological Methodology 1969, San Francisco, Jossey Bass, 1971. Boudon, R., "A propos d'un livre imaginaire", introducción de Lazarsfeld, 1970. Bulmer, M., The Chicago School of Sociology, Chicago, The University of Chicago Press, 1986.

Costner, H.L., "Research Methodology in Sociology", en Borgatta E.F. y Cook, K.S. (eds.), The Future of Sociology, Newbury Park, Sage, 1988, p. 42-56.

Coreth, E., Cuestiones fundamentales de hermeneútica, Barcelona, Herder, 1972.

De Landsheere, G., La recherche en éducation dans le monde, Paris, PUF, 1986.

Gage, N.L., "The Paradigm Wars and their Aftermath. A "Historical" Sketch of

Research on Teaching since 1989", Educational Researcher, vol.18 (oct.1989), núm. 7, p. 4-10.

Gibaja, R.E., "Acerca del debate metodológico en la investigación educacional", La Educacion. Revista Interamericana de desarrollo educativo, año XXXII (1988), núm. 103, p. 81-89. 
"Papers": Revista de Sociologia

Guba, E. (ed.), The Paradigm Dialog, Newbury Park, Sage, 1990.

Keats, J.A., "Measurement in Educational Research", en Keeves, 1988.

Keeves, J.P. (ed.), Educational Research, Methodology and Measurement: An Intemational Handbook, Oxford-N.Y., Pergamon Press, 1988.

Kendall, M.G., "Estadística: Histotia del Método Estadístico", en Silld, D.L. (ed.), Enciclopedia Internacional de las Ciencias Sociales, vol. 4, Madrid, Aguilar, 1974, p. 404-411.

Lazarsfeld, P.F., "Notes sur l'histoire de la quantification en sociologie: les sources, les tendances, les grands problèmes", Philosophie des sciences sociales, Paris, Gallimard, 1970, p. $75-162$.

Mardones, J.M. y Ursua, N. (eds.), Filosofia de las ciencias bumanas y sociales, BarceIona, Fontamara, 1982.

Renard, M. y Baker, P.M., "Social Science for the 1990's: A New Synthesis of Qualitative Methods and Environmental Ethics", Footnotes, vol.18 (nov. 1990), núm. 9, p. 18.

Reason, P. y Rowan, J. (eds.), Human Inquiny, N.Y., John Wiley \& Sons, 1981. Rist, R.C., On the relations among educational research paradigms: From Desdain to Detente, Washington, NIE, 1977. "Blitzkrieg Ethnography: On the transformation of a Method into a Movement", Educational Researcher, vol. 9 (marzo 1980), núm. 2.

Schuessler, K.F. (ed.), Sociological Methodology 1979, San Francisco, Jossey Bass, 1978.

Seiffert, H., Introducción a la teoria de la ciencia, Barcelona, Herder, 1977 (la edición original alemana fue publicada en tres volúmenes en 1969, 1970 y 1971). 